\title{
Extreme bradycardia during microsurgery of the larynx
}

\author{
Tsvetomir Marinov, MD, PhD \\ Department of Anesthesiology and Intensive Care \\ University Hospital "Queen Giovanna" - ISUL \\ Medical University - Sofia
}

\begin{abstract}
Introduction: Successful anesthetic management of microlaryngeal cases requires a high degree of cooperation with the surgeon, a reciprocal understanding of the potential problems, and adequate preparation on both sides to meet the anticipated challenges that may arise. Case description: A 55-year-old male patient (weight $104 \mathrm{~kg}$, height $176 \mathrm{~cm}$ ), ASA III, was admitted to our hospital for microlaryngeal surgery because of tumor formation of the larynx. The preoperative evaluation revealed no significant personal or family history for adverse reaction to anesthetics. A general anesthesia was scheduled for this patient. Fentanyl $0.3 \mathrm{mg}$, atropine $0.5 \mathrm{mg}$, propofol 220 $\mathrm{mg}$ and succinylcholine $130 \mathrm{mg}$ were administered for anesthesia induction. After intubation $2.5 \%$ sevoflurane was used for anesthesia maintenance. After endotracheal intubation was performed surgeon tried to put on the tube of Kleinsasser. During this procedure the heart rate of the patient extremely dropped from 85 to 25 beats per minute. Discussion: Performance of conventional and operative direct laryngoscopy, supraglottic tissue distention, and laryngeal stimulation elicit intense cardiovascular responses, resulting in tachycardia, arterial and pulmonary hypertension, and arrhythmias. Although these responses are usually short lived, myocardial ischemia and compromise of cerebral circulation may occur in high-risk patients, resulting in adverse outcomes.

Conclusion: There is a risk of severe bradycardia after putting on the tube of Kleinsasser during microlaryngeal surgery, which can cause hemodynamic instability and even cardiac arrest if not corrected immediately.

Key words: bradycardia, microsurgery of the larynx, tube of Kleinsasser
\end{abstract}

\section{Introduction}

Successful anesthetic management of microlaryngeal cases requires a high degree of cooperation with the surgeon, a reciprocal understanding of the potential problems, and adequate preparation on both sides to meet the anticipated challenges that may arise ${ }^{1,2}$. Thorough appreciation by the anesthesiologist of the complexity of the upper airway anatomy, the pathologic process involved, and all steps of the surgical procedure is necessary for devising a rational anesthetic plan and maintaining a good working relationship with the surgeon ${ }^{3}$. The expert ability to safely share the patient's airway with the surgeon, in conjunction with an intimate knowledge of possible immediate intraoperative and early postoperative complications of laryngeal surgery, greatly contributes to safe patient management in the perioperative period.

Many patients presenting for laryngeal surgery have a long history of heavy smoking and drinking ${ }^{4}$. Many of them are elderly and have cardiovascular disease. Appropriate diagnostic tests are indicated for them as part of the preoperative work-up. There are a few reports about bradycardia or asystole caused by direct laryngoscopy ${ }^{5,6}$. Transient increase in heart rate and mean arterial pressure commonly occur during manipulation of the airway via direct laryngoscopy. This phenomenon is understood to be due to a sympathetic nervous system reflex causing an increase in plasma catecholamines. Rarely, severe bradycardia and possible asystole can occur following laryngoscopy. 
One factor in the occurrence of this phenomenon is the vagal reflex. Activation of afferent parasympathetic nerve fibers during stimulation of the lower pharynx, larynx, trachea, and epiglottis may result in bradycardia and asystole ${ }^{7}$. Excessive vagal activity, which causes severe bradycardia and hypotension, can be life threatening. The trigger can be painful stimulation of the bronchial, pharyngeal, laryngeal, or esophageal mucosa.

\section{Case report}

It is reported in the case extreme bradycardia in a male patient presenting for microsurgery of the larynx.

A 55-year-old male patient (weight $104 \mathrm{~kg}$, height $176 \mathrm{~cm}$ ), ASA III, was admitted to our hospital for microlaryngeal surgery because of tumor formation of the larynx. The preoperative evaluation revealed no significant personal or family history for adverse reaction to anesthetics. A general anesthesia was scheduled for this patient. Fentanyl 0.3 $\mathrm{mg}$, atropine $0.5 \mathrm{mg}$, propofol $220 \mathrm{mg}$ and succinylcholine $130 \mathrm{mg}$ were administered for anesthesia induction. After intubation $2.5 \%$ sevoflurane was used for anesthesia maintenance. Ventilation was performed through endotracheal tube № 6.5. Mechanical ventilation was started using a ventilator (Drager Fabius Plus) in the volume control mode. A tidal volume of $650 \mathrm{ml}$, a respiratory rate of 11 per minute and an inspiratory: expiratory ratio of 1:2 was used. Normocarbia was maintained (end tidal carbon dioxide 34-38 $\mathrm{mmHg}$ ). Adequate ventilation was judged by end tidal carbon dioxide $\left(\mathrm{ETCO}_{2}\right)$ and expired tidal volume. Standard intraoperative monitoring was applied (non invasive blood pressure at 5 minutes interval, ECG, pulse oximetry and capnography).

After endotracheal intubation was performed surgeon tried to put on the tube of Kleinsasser, which is needed for visualization of the structures of the larynx. During this procedure the heart rate of the patient extremely dropped from 85 to 25 beats per minute. The surgeon was told to remove the tube and additional $0.5 \mathrm{mg}$ of atropine was administered by the anesthesiologist. After that the heart rate of the patient elevated to 90 beats per minute. The surgeon made second attempt to put on the tube of Kleinsasser, which was followed by another drop in heart rate of the patient this time to 47 beats per minute. Heart rate of the patient during the operation was between 47-53 beats per minute. After the end of the operation when the tube of Kleinsasser was removed the heart rate of the patient elevated to $85-92$ beats per minute.

Extubation was performed when the patient restores reflexes and spontaneous breathing with tidal volume of $5 \mathrm{ml} / \mathrm{kg}$. Discharge criteria from the operating room were normal respiratory pattern, adequate tidal volume and $\mathrm{SpO}_{2} \geq 98 \%$, and appropriate response to verbal stimuli, then patient was transferred to post anesthetic care unit.

\section{Discussion}

This case report illustrates that although the tip of the curved blade of the direct laryngoscope is positioned at the vallecula (between the base of the tongue and the pharyngeal surface of the epiglottis) during tracheal intubation, the blade tip of the suspension laryngoscope lifts the laryngeal surface of the epiglottis or supraglottic area during surgery. The sensory distribution of the vagus nerve is more abundant in the supraglottic area and the laryngeal surface of epiglottis than at the base of the tongue and in the pharyngeal surface of epiglottis. Therefore, suspension laryngoscopy can be said more vagotonic than curvedblade direct laryngoscopy.

The glossopharyngeal sensory nerve fibers innervated in the nasopharynx, the caudal half of the soft palate, the caudal one third of the tongue, the vallecula, the pharyngeal surface of epiglottis, and the pharyngeal wall. Internal branch of the superior laryngeal nerve originated from the vagus nerve innervated in the laryngeal surface of the epiglottis, the laryngeal vestibulum, posterior wall of the glottis, caudal aspect of the vocal fold, subglottis and joining with the recurrent laryngeal nerve ${ }^{8}$.

Performance of conventional and operative direct laryngoscopy, supraglottic tissue distention, and laryngeal stimulation elicit intense cardiovascular responses, resulting in tachycardia, arterial and pulmonary hypertension, and arrhythmias 9. Although these responses are usually short lived, myocardial ischemia and compromise of cerebral circulation may occur in high-risk patients, resulting in adverse outcomes ${ }^{10}$. Anesthetic technique should ensure a stable plane of anesthesia, nonstimulating emergence from anesthesia, a rapid return of consciousness, and protective airway reflexes, and it should facilitate quick discharge of patients, because most of these surgical procedures are done on an outpatient basis.

Some induction drugs also induce bradycardia which may be accompanied by vasodilatation which is also a feature of certain pathologies, which influence the progression to 'unstable' bra- 
dycardia, which does not respond to re-oxygenation and a pause in laryngoscopy. Preintubation atropine diminishes the overall incidence of stable bradycardia during routine anesthesia. However, clinical studies of critical care intubation show that atropine does not prevent all episodes of bradycardia and specifically cannot affect vasodilatation ${ }^{11}$. The essential requirements for precision microlaryngeal surgery and optimal preservation of function include a clear and still surgical field, absence of patient movement, and allocation of sufficient time to carefully complete the procedure in an unhurried manner. There is a study conducted by Marinov et al. among 200 patients who underwent microlaryngeal surgery in the Department of ENT
Surgery; University Hospital "Queen Giovanna"ISUL. It shows that $40 \%$ of the patients had pulse rate between $45-60$ beats per minute during putting on the tube of Kleinsasser, $29 \%$ of them had pulse rate $>60$ beats per minute during putting on the tube of Kleinsasser and $31 \%$ had pulse rate $<45$ beats per minute during putting on the tube of Kleinsasser. This indicates that bradycardia is a common occurrence in this surgery ${ }^{12,13}$.

\section{Conclusion}

There is a risk of severe bradycardia after putting on the tube of Kleinsasser during microlaryngeal surgery, which can cause hemodynamic instability and even cardiac arrest if not corrected immediately.

\title{
References
}

1. Webster AC: Anesthesia for operations on the upper airway. Int Anesthesiol Clin 10:61-122, 1972.

2. Agosti L: Anaesthetic technique for microsurgery of the larynx. Anaesthesia 32:362-365, 1977.

3. Welty P: Anesthetic concerns and complications during suspension microlaryngoscopy procedures. CRNA 3:113-118, 1992.

4. Rees L, Mason RA: Advanced upper airway obstruction in ENT surgery. Br J Anaesth CEPD Rev 2:134-138, 2002.

5. Cheong KF, Manivannan GK, Yau GH. Asystole following laryngoscopy and endotracheal intubation: a case report. Ann Acad Med Singapore. 1996;25:283-285.

6. Mizuno J, Mizuno S, Ono N, Yajima C, Arita H, Hanaoka K. Sinus arrest during laryngoscopy for induction of general anesthesia with intravenous fentanyl and propofol. Masui. 2005;54:1030-1033.

7. Sutera PT, Smith CE. Asystole during direct laryngoscopy and tracheal intubation. J Cardiothorac Vasc Anesth. 1994;8:79-80.

8. Yoshida Y, Tanaka Y, Hirano M, Nakashima T. Sensory innervation of the pharynx and larynx. Am J Med. 2000;108(Suppl 4a):51S-61S.

9. Kaplan JD, Schuster DP: Physiologic consequences of tracheal intubation. Clin Chest Med 12:425-432, 1991.

10. Siddiqui N, Katznelson R, Friedman Z: Heart rate/blood pressure response and airway morbidity following tracheal intubation with direct laryngoscopy, GlideScope and Trachlight: A randomized control trial. Eur J Anaesthesiol 26:740-745, 2009

11. Jones, Peter, Stéphane Dauger, and Mark J. Peters. "Bradycardia during critical care intubation: mechanisms, significance and atropine." Archives of disease in childhood 97.2 (2012): 139-144.

12. Sapundzhiev, N., Stelianova, T., Gencheva, M., Platikanov, V. General endotracheal anesthesia with intermittent apnea technique in microlaryngeal surgery. Anaesthesiology and Intensive Care, 2007, 34(5), pp. 3-6.

13. Marinov, T., Belitova, M., Popov, T., Nizamova, N., Rangachev, J., \& Popova, D. (2020). Hemodynamic complications during microlaryngeal surgery. International Bulletin of Otorhinolaryngology, 16(3), 35-38.

Reviewers: Assoc. prof. Dr. Alexander Vulkov Vulkov / Prof. Dr. V. Platikanov

\author{
Correspondence to: \\ Tsvetomir Marinov, MD, PhD \\ Department of Anesthesiology and Intensive Care \\ University Hospital "Queen Giovanna”- ISUL \\ Byalo More Str 8 \\ Medical University - Sofia \\ E-mail: ts.marinov@abv.bg
}

